\title{
21. GALACTIC CONTINUUM SOURCE COUNTS
}

\author{
T. L. WILSON
}

National Radio Astronomy Observatory, Charlottesville, Va., U.S.A.

The CSIRO $11 \mathrm{~cm}$ continuum survey of the galactic plane from $l^{\mathrm{II}}=280^{\circ}$ to $37^{\circ}$ has recently been finished. I have used this survey to compare the number of sources in various $5^{\circ}$ intervals north and south of the galactic center. The NRAO-CSIRO-MIT H 109 $\alpha$ line survey (Wilson et al., 1970) found 39 sources lying between $330^{\circ}$ and $340^{\circ}$, while the NRAO-MIT (Reifenstein et al., 1970) survey found only 14 between $20^{\circ}$ and $30^{\circ}$. If the galaxy were symmetric, one would expect roughly equal numbers. One suspects a selection effect is present, and a rough check is very worthwhile. The CSIRO survey is particularly valuable since it is the highest resolution survey covering large portions of the galaxy on both sides of the center.

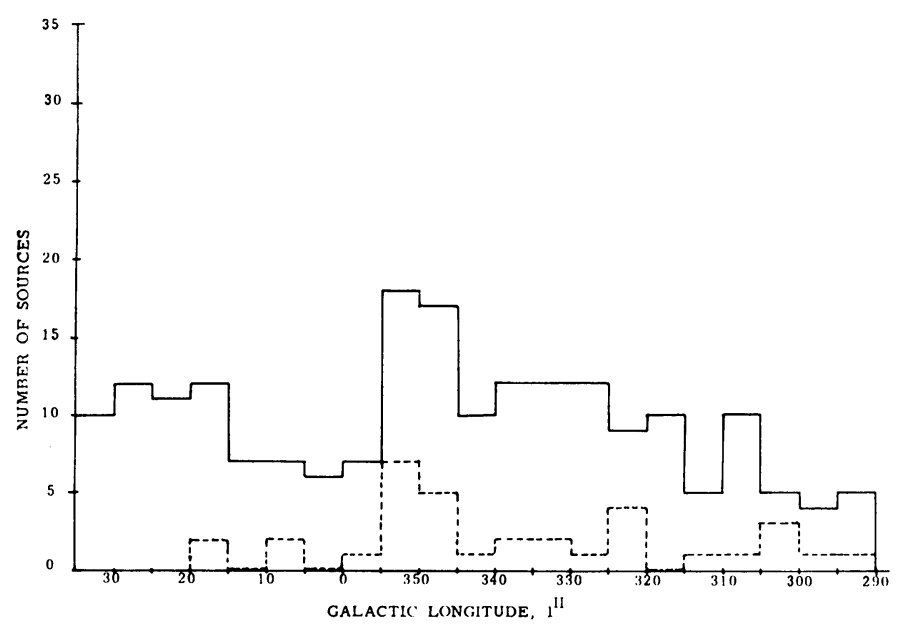

Fig. 1. Plot of number of sources per $5^{\circ}$ interval, as a function of $l^{11}$. Solid line - source number. Dotted line - number of optically identified sources.

We have included only those sources which would be present in the $\mathrm{H} 109 \alpha$ line surveys, that is those with peak brightness temperatures above $2 \mathrm{~K}$. These, averaged over $5^{\circ}$ of longitude, form the solid curve, while the dotted curve is the number of optical identifications per $5^{\circ}$ interval. The optical sources are taken from the $\mathrm{RCW}$ catalogue. The number of sources in the two zones $20^{\circ}-30^{\circ}$ and $330^{\circ}-340^{\circ}$, are roughly the same, so that one concludes that the larger numbers found in the south are due to some selection effect, probably the higher angular resolution of the $210^{\prime}$ radio telescope.

An interesting point is the large peak between $345^{\circ}$ and $355^{\circ}$. This is probably mostly due to a nearby cluster of sources, although the $\mathrm{H} 109 \alpha$ line shows that 5 sources 
possess rather high velocities. This hypothesis is supported by the somewhat higher number of optical identifications and the low radial velocity found from the recombination line surveys. The velocities are shown in Figure 2.

The average velocities in the $345^{\circ}-355^{\circ}$ zone are about $-30 \mathrm{~km} \mathrm{~s}^{-1}$, far below the maximum Schmidt model velocity $-50 \mathrm{~km} \mathrm{~s}^{-1}$ for sources outside the $4 \mathrm{kpc}$ arm at this longitude. The velocities in the $330^{\circ}-340^{\circ}$ and $20^{\circ}-30^{\circ}$ zones are much higher,

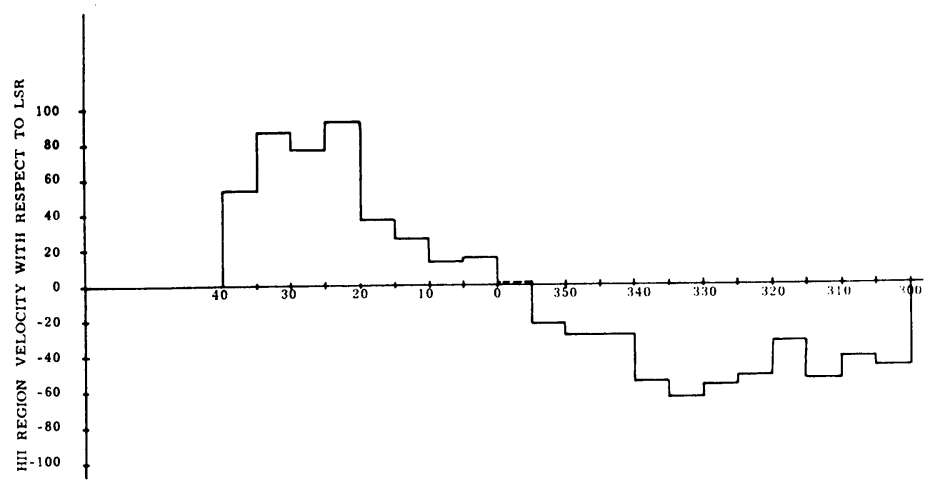

Fig. 2. H II region VLSR's averaged over $5^{\circ}$ intervals, plotted against $l$ III.

although not equal. These last two zones, between 4 and $6 \mathrm{kpc}$ from the center, contain most of the giant $\mathrm{H}$ II regions in the galaxy. They do not show up well in the $11 \mathrm{~cm}$ survey because they are quite distant and their individual sources cannot be resolved. The nearby sources bias us very strongly and only a knowledge of their distance, through their radial velocities, can tell us which are the nearby and which are the distant, significant features.

\section{Acknowledgements}

I am indebted to Dr. F. J. Kerr for valuable discussions on this subject. The NRAO is operated by Associated Universities, Inc., under contract with the National Science Foundation. The CSIRO Survey was performed by M. Beard, G. A. Day, W. M. Goss, F. J. Kerr, and B. M. Thomas. I am indebted to Mr. Day for a preliminary version of the map of the region from $6^{\circ}$ to $26^{\circ}$.

\section{References}

Reifenstein, E. C. III, Wilson, T. L., Burke, B. F., Mezger, P. G., and Altenhoff, W. J.: 1970, Astron. Astrophys. 4, 357.

Wilson, T. L., Mezger, P. G., Gardner, F. F., and Milne, D. K.: 1970, in preparation. 